\title{
Well Differentiated Malignant Hemangiopericytoma
}

National Cancer Institute

\section{Source}

National Cancer Institute. Well Differentiated Malignant Hemangiopericytoma. NCI Thesaurus. Code C9392.

A malignant hemang iopericytoma with well-differentiated morphological features. 